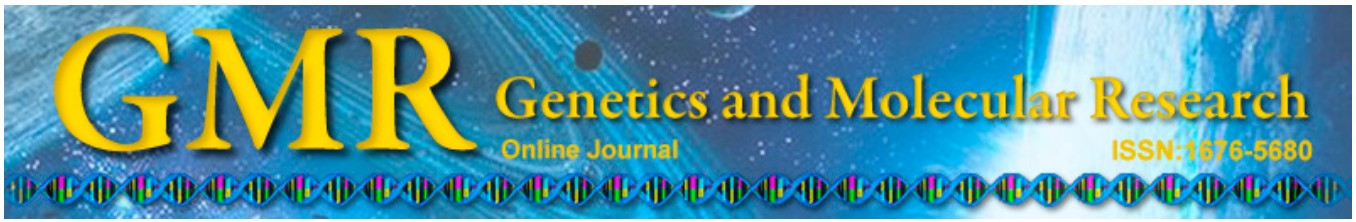

\title{
MULTINDELS-BOV: Zebu traceback method based on DNA insertion-deletion polymorphisms
}

\author{
M. Groenner-Penna ${ }^{1,2}$, E.F.D. Croce ${ }^{1}$, C.G. Pimenta ${ }^{1}$, H.M.S. Bicalho ${ }^{1}$ and \\ S.D.J. Pena ${ }^{1,2}$ \\ ${ }^{1}$ Gene-Genealógica Central de GenoTipagem de Animais, \\ Belo Horizonte, MG, Brasil \\ ${ }^{2}$ Laboratório de Genômica Clínica, Faculdade de Medicina, \\ Universidade Federal de Minas Gerais, Belo Horizonte, MG, Brasil \\ Corresponding author: S.D.J. Pena \\ E-mail: spena@dcc.ufmg.br
}

Genet. Mol. Res. 13 (4): 9171-9186 (2014)

Received October 29, 2014

Accepted November 3, 2014

Published November 7, 2014

DOI http://dx.doi.org/10.4238/2014.November.7.4

\begin{abstract}
Brazil is a major producer and exporter of beef, with a herd of approximately 210 million animals. For the meat industry, a reliable animal traceback from its origin to the consumer market is paramount. Of all available identification systems, DNA is the only one that survives the slaughterhouse and reaches the dish of the consumer. DNA polymorphisms are already used for cattle traceback, but primarily for the subspecies Bos taurus taurus. However, in Brazil, another subspecies, B. taurus indicus predominates. We describe here the development of a DNA traceback method designed primarily for $B$. taurus indicus (Zebu), without leaving B. taurus taurus aside. We used insertion/deletion (indel) polymorphisms, which have the advantage of being simple and easily automatable, since in most cases, the variable loci are biallelic. We studied 94 indels, with a difference of two or more
\end{abstract}


base pairs, in DNA pools of 60 Zebu and 60 taurine animals. A set of 22 indels with heterozygosity greater than 0.3 were selected and used to construct two multiplex PCRs. On the basis of the allelic frequency of these indels, the probability of random match was calculated to be $1.12 \times 10^{-8}$ for B. taurus indicus and $1.60 \times 10^{-6}$ for B. taurus taurus. Moreover, we estimated that an analysis would cost less than US $\$ 15.00$ per animal. Thus, this system (MULTINDELS-BOV) is perfectly suited for building large genetic databases and offering viable prospects of a national system for cattle traceback DNA in Brazil.

Key words: Cattle; Traceback; Indel; Polymorphism; Bos taurus indicus

\section{INTRODUCTION}

Brazil is one of the major producers and exporters of beef in the world, featuring a herd of approximately 210 million animals (FAOSTAT, 2011). For the beef trade, a reliable animal identification from its origin to the consumer market is of crucial importance. There are several ways to identify the animals physically, such as iron markings, earrings, subcutaneous chips, and retina reading (Lima et al., 2006; Bowling et al., 2008). Nevertheless, all these types of markings are lost in the slaughterhouse. DNA is the only tag that cannot be removed from the meat and persists up to the dish of the consumer.

Currently, single nucleotide polymorphisms (SNPs) and microsatellites are the two classes of DNA polymorphisms most commonly used for animal identification (Pascoal et al., 2005; Shackell et al., 2005; Karniol et al., 2009; Orrù et al., 2009; Allen et al., 2010). However, both present some problems: SNPs require expensive equipment for typing, and microsatellites are not optimal for multiplexing because they are multi-allelic. Another relevant fact is that current DNA traceback methods have been developed for testing animals of the Bos taurus taurus subspecies. Nevertheless, in Brazil and also some other important producing countries, B. taurus indicus predominates (Groeneveld et al., 2010), and there is a need to develop methods for traceback that are efficient for this subspecies.

Thus, in the development of a DNA traceback method for use in Brazil, we focused our attention primarily to user-friendly inexpensive molecular methods and on the subspecies B. taurus indicus (Zebu or Indicine cattle), however without neglecting the B. taurus taurus subspecies (European or taurine cattle). For the development of such method, we decided to use insertion/deletion (indel) polymorphisms, which have the advantage of being simple, rapid and easily automatable, since in most cases the variable loci have only two alleles. Moreover, the size of the amplicons can be reduced to 50-60 bp if needed, permitting the typing of degraded DNA (Bastos-Rodrigues et al., 2006).

\section{MATERIAL AND METHODS}

\section{Samples}

The samples used in this study belonged to the company Gene-Genealógica Central de GenoTipagem de Animais Ltda. which has a bank of genetic material extracted from hair 
follicles, blood, semen, and tissue of animals genotyped on the request of breeders and breed associations throughout Brazil. To assess the insertion and deletion polymorphisms in the two subspecies of cattle, DNA pools were made from 60 B. taurus indicus animals (including 10 samples of each of the races Gyr, Nellore, Guzerat, Brahman, Sindhi, and Tabapuan) and 60 B. taurus taurus animals (including 20 samples of each of the races Holstein, Jersey, and Angus). These pools of DNA were used in PCR for the identification of polymorphic loci and the determination of allelic frequencies.

\section{DNA extraction}

Genomic DNA was extracted from four carefully selected intact hair follicles, using the proteinase $\mathrm{K}$ method $(50 \mu \mathrm{L}$ Madisen solution, $0.1 \mathrm{M}$ Tris-HCl, $\mathrm{pH}$ 8.0, $0.04 \mathrm{M}$ EDTA, $\mathrm{pH}$ $8.0,1 \mathrm{M} \mathrm{NaCl}, 0.2 \% \mathrm{SDS}$, and $2 \mu \mathrm{L} 20 \mathrm{mg} / \mathrm{mL}$ proteinase $\mathrm{K}$, with incubation for $3 \mathrm{~h}$ at $56^{\circ} \mathrm{C}$ ), giving an efficiency of $30 \mathrm{ng} / \mathrm{mL}$. The procedure for extracting DNA from blood and semen was performed with $5 \%$ Chelex $100{ }^{\circledR}$. The DNA samples were stored at room temperature.

\section{Insertion-deletion polymorphisms (indels)}

For this study, 75 indels were selected from dbSNP, from NCBI (National Center for Biotechnology Information) because they showed a difference of 2 or more bp between alleles, which facilitated the detection of the polymorphism. We also examined the relevant literature and found another 21 indels (Hills et al., 2003; Sander et al., 2004; Nakatsu et al., 2004; Siadkowska et al., 2006; Ferraz et al., 2006; Cargill and Womack, 2007; Sasazaki et al., 2007; Hoashi et al., 2007; Seabury et al., 2008). Since our primer design did not work properly for two indels (which would be Ibov68 and Ibov69 in Tables 1 and 2), they were discarded and thus, altogether, 94 indels previously reported as polymorphic in B. taurus taurus were analyzed in the Zebu animals (Table 1).

\section{Analysis of indels}

The primers used for amplification of the indel region were designed in the PrimerBLAST program (Ye et al., 2012) and are shown in Table 2. PCR was carried out with: 0.36 $\mu \mathrm{L} 1 \mu \mathrm{M}$ primer F, $0.18 \mu \mathrm{L} 10 \mu \mathrm{M}$ primer R, $0.18 \mu \mathrm{L} 10 \mu \mathrm{M}$ M13-FAM or M13-HEX, $6.8 \mu \mathrm{L}$ universal pre-mix (1.32X Taq DNA polymerase buffer, mix of dATP, dCTP, dTTP, and dGTP in $0.36 \mu \mathrm{M} 4.63 \mu \mathrm{M} \mathrm{MgCl}_{2}$ ), $0.2 \mu \mathrm{L} \mathrm{Taq}$ DNA polymerase, $0.28 \mu \mathrm{L} \mathrm{H}_{2} \mathrm{O}$, and $1.0 \mu \mathrm{L} 30 \mathrm{ng} / \mu \mathrm{L}$ DNA or $\mathrm{H}_{2} \mathrm{O}$. A stepdown PCR program was used in a thermocycler: $95^{\circ} \mathrm{C}$ for $1 \mathrm{~min}, 2$ cycles at each annealing temperature, $95^{\circ} \mathrm{C}$ for $45 \mathrm{~s}$, touchdown from 61 to $53^{\circ} \mathrm{C}$ for $45 \mathrm{~s}$ and $72^{\circ} \mathrm{C}$ for $1 \mathrm{~min} ; 25$ cycles of $94^{\circ} \mathrm{C}$ for $45 \mathrm{~s}, 53^{\circ} \mathrm{C}$ for $45 \mathrm{~s}$, and $72^{\circ} \mathrm{C}$ for $1 \mathrm{~min}$. The amplicons were detected on the MegaBACE $1000^{\circledR}$ following the guidelines of the manufacturer (GE). Reading the results that were generated in MegaBACE $1000^{\circledR}$ was performed with MegaBACE Fragment Profile 1.2 from Amersham Biosciences (GE). Starting with PCR using 96 primer pairs, tests were performed pooling DNA from 60 Zebu and 60 taurine animals. The height of the curves obtained for each amplicon was used as a reference for quantification of each indel allele and for calculating the frequency of each allele. 
Table 1. Indel loci known to be polymorphic in Bos taurus taurus analyzed for the estimation of variability in Bos taurus indicus.

\begin{tabular}{|c|c|c|c|}
\hline Indel name & Indel size & dbSNP rs id & Reference \\
\hline IBov01 & 3 & rs29003887 & \\
\hline IBov02 & 3 & rs29002112 & \\
\hline IBov03 & 3 & rs17870616 & \\
\hline IBov04 & 3 & rs29002187 & \\
\hline IBov05 & 3 & rs29003113 & \\
\hline IBov06 & 3 & rs29003131 & \\
\hline IBov07 & 3 & rs 29002704 & \\
\hline IBov08 & 3 & rs29003043 & \\
\hline IBov09 & 3 & rs29003907 & \\
\hline IBov10 & 4 & rs29004154 & \\
\hline IBov11 & 4 & rs 17870234 & \\
\hline IBov12 & 4 & rs29002299 & \\
\hline IBov13 & 4 & rs29002184 & \\
\hline IBov14 & 4 & rs29003128 & \\
\hline IBov15 & 4 & rs29001780 & \\
\hline IBov16 & 4 & rs29003986 & \\
\hline IBov17 & 4 & rs29002819 & \\
\hline IBov18 & 4 & rs 29002278 & \\
\hline IBov19 & 4 & rs 29002520 & \\
\hline IBov20 & 4 & rs29003512 & \\
\hline IBov21 & 4 & rs29003233 & \\
\hline IBov22 & 5 & rs 17870560 & \\
\hline IBov23 & 5 & rs41257489 & \\
\hline IBov24 & 5 & rs 29002827 & \\
\hline IBov25 & 5 & rs41255797 & \\
\hline IBov26 & 6 & rs 17870558 & \\
\hline IBov27 & 6 & rs 29003033 & \\
\hline IBov28 & 9 & rs 17870280 & \\
\hline IBov29 & 9 & rs 29002813 & \\
\hline IBov30 & 9 & rs41257431 & \\
\hline IBov31 & 10 & rs41257476 & \\
\hline IBov32 & 10 & rs 29003426 & \\
\hline IBov33 & 12 & rs 29004129 & \\
\hline IBov34 & 13 & rs41255830 & \\
\hline IBov35 & 15 & rs 29002795 & \\
\hline IBov36 & 15 & rs41257558 & \\
\hline IBov37 & 15 & rs29003697 & \\
\hline IBov38 & 18 & rs29003341 & \\
\hline IBov39 & 19 & rs29002493 & \\
\hline IBov40 & 22 & rs29002500 & \\
\hline IBov41 & 25 & rs 55617435 & \\
\hline IBov42 & 3 & rs 17873919 & \\
\hline IBov43 & 3 & rs 17874005 & \\
\hline IBov44 & 4 & rs 17873113 & \\
\hline IBov45 & 4 & rs 17873935 & \\
\hline
\end{tabular}

IBov46

IBov47

IBov48

IBov49

IBov50

IBov51

IBov52

IBov53

IBov54

IBov55

IBov56

IBov57

IBov58

IBov59

IBov60

IBov61

IBov62

IBov63

IBov64

IBov65

argill and Womack, 2007

Cargill and Womack, 2007

Cargill and Womack, 2007

Cargill and Womack, 2007

Cargill and Womack, 2007

Cargill and Womack, 2007

Hills et al., 2003

Sander et al., 2004

Sander et al., 2004

Hills et al., 2003

Sasazaki et al., 2007

Siadkowska et al., 2006

Nakatsu et al., 2004

Hoashi et al., 2007

Seabury et al., 2007

Hills et al., 2003

Hills et al., 2003

Hills et al., 2003

Hills et al., 2003

Ferraz et al., 2006

Continued on next page

Genetics and Molecular Research 13 (4): 9171-9186 (2014) 


\begin{tabular}{|c|c|c|c|}
\hline Indel name & Indel size & dbSNP rs id & Reference \\
\hline Ibov66 & 2 & rs41257498 & \\
\hline Ibov67 & 2 & rs41257516 & \\
\hline Ibov70 & 2 & rs29002108 & \\
\hline Ibov71 & 2 & rs41255863 & \\
\hline Ibov72 & 2 & rs17870457 & \\
\hline Ibov73 & 2 & rs29004160 & \\
\hline Ibov74 & 2 & rs29002345 & \\
\hline Ibov 75 & 2 & rs29002207 & \\
\hline Ibov76 & 2 & rs29002409 & \\
\hline Ibov77 & 2 & rs29002264 & \\
\hline Ibov 78 & 2 & rs17870298 & \\
\hline Ibov79 & 2 & rs29004149 & \\
\hline Ibov80 & 2 & rs29004145 & \\
\hline Ibov81 & 2 & rs29004094 & \\
\hline Ibov82 & 2 & rs29003872 & \\
\hline Ibov83 & 2 & rs29002731 & \\
\hline Ibov84 & 2 & rs41255780 & \\
\hline Ibov85 & 2 & rs29002611 & \\
\hline Ibov86 & 2 & rs29003044 & \\
\hline Ibov87 & 2 & rs 17870220 & \\
\hline Ibov88 & 2 & rs 17870219 & \\
\hline Ibov89 & 2 & rs29004134 & \\
\hline Ibov90 & 2 & rs29003567 & \\
\hline Ibov91 & 2 & rs17871656 & \\
\hline Ibov92 & 2 & rs29002556 & \\
\hline Ibov93 & 2 & rs29002550 & \\
\hline Ibov94 & 3 & rs 108452982 & \\
\hline Ibov95 & 3 & rs 110022434 & \\
\hline Ibov96 & 2 & rs68268241 & \\
\hline
\end{tabular}

\section{Multiplex PCR}

The 22 indel loci with heterozygosity closest to 0.5 were selected for multiplex PCR amplification. New primers were designed for multiplexing using the muPlex software (Rachlin et al., 2005) (Table 4). These primers were grouped into two multiplexes of 11 indels each for use in two PCR analyses (Table 5). Two primer mixes were made according to the following conditions: $2.3 \mu \mathrm{L} 1 \mu \mathrm{M}$ of each primer F, $2.3 \mu \mathrm{L} 10 \mu \mathrm{M}$ of each primer R, 25.3 $\mu \mathrm{L} 10 \mu \mathrm{M}$ M13-FAM or M13-HEX. From these primer mixes, we used $1 \mu \mathrm{L}$ in the PCR with $6.8 \mu \mathrm{L}$ universal pre-mix (1.32X Taq DNA polymerase buffer, mix of dATP, dCTP, dTTP, and dGTP in $0.36 \mu \mathrm{M} 4.63 \mu \mathrm{M} \mathrm{MgCl}_{2}$ ), $0.2 \mu \mathrm{L} \mathrm{Taq}$ DNA polymerase, $0.28 \mu \mathrm{L} \mathrm{H}_{2} \mathrm{O}$, and 1.0 $\mu \mathrm{L}$ DNA or $\mathrm{H}_{2} \mathrm{O}$. The thermocycler program used was the same as described before. The amplified fragments were analyzed as described previously.

\section{Indel analysis in the Structure program}

The Structure program, version 2.3.4 (Pritchard et al., 2000), was used to determine if the indel panel could identify the Bos subspecies. $\mathrm{K}=2$ ( $\mathrm{k}$ is the number of populations) was used. Although Zebu cattle also have some taurine ancestry (Kumar et al., 2003), this is relatively small and can be ignored for this purpose. The admixture model was used with correlated allele frequencies. Each run consisted of 50,000 burn-in steps, followed by 250,000 Markov chain Monte Carlo interactions. 
Table 2. PCR primers for amplification of indels in Bos taurus indicus.

\begin{tabular}{|c|c|}
\hline Primer name & Primer sequence (5'-3') \\
\hline Ibov01F & TGGTCTGCCTTACTCTGGTC \\
\hline Ibov01R & GTCATACTGTGTAGCCAATAGCC \\
\hline Ibov02F & CTATCCCAAAGCCTCAGATCC \\
\hline Ibov02R & GTCATTGGCTGTTGCTCTTC \\
\hline Ibov03F & CTTCTAGTATCTAGTGGAGGCCA \\
\hline Ibov03R & CAGCATGGCGTAGCTCTCATAC \\
\hline Ibov04F & CTTGGGTGAAGGGGAACTTCG \\
\hline Ibov04R & GGGGCTGCAAATAAAGACAGAC \\
\hline Ibov05F & CGCTATGAATGAGGATGTAGTCC \\
\hline Ibov05R & ССТССССТТСАСАСТТТСТАСТАС \\
\hline Ibov06F & СТCTACTTGATTGGAGCTGGAAG \\
\hline Ibov06R & CCCCATGCTTTCTGTGAGAG \\
\hline Ibov07F & CACTAACCTTAGCTCCTCTCAC \\
\hline Ibov07R & GCTTTTCCAAGGACTCACAC \\
\hline Ibov08F & CTAATCCAGTCAGTGTACCTCC \\
\hline Ibov08R & CGCTGAACAGTATTCGAGTTAC \\
\hline Ibov09F & CACTACTCCACCTTGTATACCTTG \\
\hline Ibov09R & GACAGAAAGGGAATCGCTGAG \\
\hline Ibov10F & GGCCATAAAACAGATTGTCTGAG \\
\hline Ibov10R & CCAGAGAGATTGGCTAGTATGTC \\
\hline Ibov11F & CTAGCAGAGGAGATGCCACG \\
\hline Ibov11R & GCCTCAGAGCCTCACACATG \\
\hline Ibov12F & GCTGTTAGCCATCAGGCAAG \\
\hline Ibov12R & GGCATAGACTAATTTCTGTCTGCATC \\
\hline Ibov13F & CTGTTTAACAGTTTTGGGAGCTG \\
\hline Ibov13R & GTATACAAGGCAATGAGGCC \\
\hline Ibov14F & GTCAAGTCTAGCCTGTGCTG \\
\hline Ibov14R & GGATGCTACCATTTGGTCAG \\
\hline Ibov15F & GAAGAGCAATAGCCCAGTGC \\
\hline Ibov15R & GCTGAGGAATTCTCTGATTATCTCC \\
\hline Ibov16F & CCTTACAGCTTCTTCTAAATGGAG \\
\hline Ibov16R & GATTGCACCTTACTATCAGAAAGG \\
\hline Ibov17F & CAGTCTGTTCTCACCTTCCAG \\
\hline Ibov17R & CTGCCAGAAGGTAATGAGACG \\
\hline Ibov18F & CCATTGGCACTGACTCTAAGTC \\
\hline Ibov18R & GTTTGGTCTTCAGGTCAGAAAG \\
\hline Ibov19F & CGCCAATGCAAGAGATGTAAG \\
\hline Ibov19R & CСTCATTCCTGGGGAAACTC \\
\hline Ibov20F & CACGCACTGCAACAACTAAG \\
\hline Ibov20R & GAGGACTGAAAGAACTTCCCC \\
\hline Ibov21F & TCTGCATTGCTAGAGATGGAAG \\
\hline Ibov21R & GGGTCAAAAACAGATTCCAGAG \\
\hline Ibov22F & GGAGGGAGACCTCAATTTCAC \\
\hline Ibov22R & СTCTCTGTGTTTGTCCTGAGG \\
\hline Ibov23F & CAGGGTGAAGGTCACAGAAC \\
\hline Ibov23R & GCCCCTTTTGTACAGATGGAG \\
\hline Ibov24F & AGAAACTGCAAAAGTACAAACAAG \\
\hline Ibov24R & TGAAGTCGCTCAGTCGTGTC \\
\hline Ibov25F & GGAGCTGAGACTGTGACTG \\
\hline Ibov25R & GGGAACCATGAATTCTGCTG \\
\hline Ibov26F & CCAATGAAGGTGAAGCGTCC \\
\hline Ibov26R & CTGAGGCTGCTCTAAGAGTTC \\
\hline Ibov27F & CAGGAGAGCTGATTTCTAGTTCTG \\
\hline Ibov27R & CTATTCATAGTGGAGCTGGGC \\
\hline Ibov $28 \mathrm{~F}$ & GTAGAGAGACCCTGAAGCAGC \\
\hline Ibov28R & ACAGCCTTGCTGGTGACATC \\
\hline Ibov29F & CACCTAGCGGTGCAACATATC \\
\hline Ibov29R & GGCATTGACTCCATCTGCAGTA \\
\hline Ibov30F & TGAGATGACCCTCTTTGAAGGAAC \\
\hline Ibov30R & GGATGGCTGCTTGGCTTATAG \\
\hline Ibov31F & GCTAATTCTCTTGACTTGCAGGC \\
\hline Ibov31R & CGTGGTGCCTCCGTTCTATG \\
\hline Ibov32F & GATACCGACAGCAGAGAGAGG \\
\hline
\end{tabular}

Continued on next page

Genetics and Molecular Research 13 (4): 9171-9186 (2014)

CFUNPEC-RP www.funpecrp.com.br 
Table 2. Continued.

\begin{tabular}{|c|c|}
\hline$\underline{\text { Primer name }}$ & Primer sequence $\left(5^{\prime}-3^{\prime}\right)$ \\
\hline Ibov32R & TCCTAAACGCTTTGTCATCCTGT \\
\hline Ibov33F & GAAAGACATAATCAGGCTCTCCAG \\
\hline Ibov33R & CTAGGGTCTTAATTGTATGTTCCTG \\
\hline Ibov34F & GTAGAAGTTCTACCACTGCCAG \\
\hline Ibov34R & GCCACACCTATTGCTCTATATGAA \\
\hline Ibov35F & СТССССТTCTTTCAGGACAC \\
\hline Ibov35R & CCAGAGAGAGACAAGCAAGC \\
\hline Ibov36F & GCAGAGGCTGGTCACCTAAG \\
\hline Ibov36R & CCATTCCAGGCACACCTACT \\
\hline Ibov37F & GGCAGAGGAGAGTCAAGTCC \\
\hline Ibov37R & CAGAGGCTGGTCACCTAAGG \\
\hline Ibov38F & TAGCTCTGAGAGAGAGGAAAAGG \\
\hline Ibov38R & TGTAGGGACACCACTTTTCTGC \\
\hline Ibov39F & GAAGGACCAATCCTCCCCTG \\
\hline Ibov39R & CCAAGCAATCCTGAGTCCTG \\
\hline Ibov40F & GAGAGCACACATGTCTTCTGTC \\
\hline Ibov40R & CTGTGGTTGTCCTCTGGTGTC \\
\hline Ibov41F & CTGGCAGGCAGATTCGTAAC \\
\hline Ibov41R & GGGTGATTTGTGGTAGGGTTTC \\
\hline Ibov $42 \mathrm{~F}$ & GAGGTTCACAATAGGATTCACGC \\
\hline Ibov42R & CGGTAAGCAACCGAATGAAGC \\
\hline Ibov43F & CACACCATGAAATTCCAGCTC \\
\hline Ibov43R & CCATGCTGAAGACACAGGAG \\
\hline Ibov44F & CAGAACAAGAGAGAAGCTGGTC \\
\hline Ibov44R & GCTGTGTGTGAGTCAGCTATC \\
\hline Ibov45F & GCTCACTGTAAATGGTTCGCTC \\
\hline Ibov45R & TTTGTGATTTCATGGCAGGG \\
\hline Ibov46F & GAACTCTCCTCTCTTGCATCCTTC \\
\hline Ibov46R & GGTATCTAAGGCCAAGGGATGC \\
\hline Ibov47F & GGCAACCAGTGTTAGAATTTGG \\
\hline Ibov47R & CTTAGCCACTAGATCGCCAG \\
\hline Ibov48F & GTGATGGGCTGGTAACAGAG \\
\hline Ibov48R & CAGTTTAGTCGCTCAGTCGTG \\
\hline Ibov49F & CTGAGCGACTAAACTGAATCTAATC \\
\hline Ibov49R & GAAGGCAGGAGAGAAGGAATC \\
\hline Ibov $50 \mathrm{~F}$ & CCTATTTCAGGCCTTTTGTTACC \\
\hline Ibov50R & GTACGTTTTACCCTTTCACAACTG \\
\hline Ibov $51 \mathrm{~F}$ & CTCACCCATGTGTTGCCAGAAGAGC \\
\hline Ibov51R & CCACATCCTCTACTTGCCACCCTTC \\
\hline Ibov $52 \mathrm{~F}$ & GAATCGGATTGGTGGGAGGC \\
\hline Ibov52R & GGCTAGATTCCTACACACCACC \\
\hline Ibov53F & GTTCTGCACTGTTATTACTGGC \\
\hline Ibov53R & CCAGGGGATTTACATGACCTAG \\
\hline Ibov54F & CAGTACACCTGATTTTCAAGTCC \\
\hline Ibov54R & GTAGGCCAAAGAGTTGGACAG \\
\hline Ibov $55 \mathrm{~F}$ & TGGAGGCAACCGTTATCCAC \\
\hline Ibov55R & GGCTTACTGGGTTTGTTCCATTG \\
\hline Ibov $56 \mathrm{~F}$ & ATGGGGCCAACAGAATCTTAG \\
\hline Ibov56R & CATGGTAAGAGGAGCCCAAG \\
\hline Ibov $57 \mathrm{~F}$ & CTGGGTGGAGCAGTGAACAC \\
\hline Ibov57R & GTGGGCTTGTCTGTTCAGATCA \\
\hline Ibov $58 \mathrm{~F}$ & GATTTCCTTCGATGCTTCATGC \\
\hline Ibov58R & CATTCACAAGTATGGCACTCATG \\
\hline Ibov59F & CACAACGCCATCGAGAAACGCTAC \\
\hline Ibov59R & GTGGGAGGGAGATGGCACAAGTG \\
\hline Ibov60F & CCCAGATATGGAAACTGAACCAG \\
\hline Ibov60R & GCTGGAAGGTAAGGGAGGAG \\
\hline Ibov61F & CAGACACGTATCTCCCTGTAG \\
\hline Ibov61R & СТCATCTTCCTGCCATCTCTG \\
\hline Ibov62F & GAGTGGGTGCAATCTGATGAC \\
\hline Ibov62R & ATTCCTGACCAAGGGAAGACC \\
\hline Ibov63F & CAGTCCTGAGTCAGGCAATG \\
\hline
\end{tabular}

Continued on next page 
Table 2. Continued.

\begin{tabular}{|c|c|}
\hline Primer name & Primer sequence $\left(5^{\prime}-3^{\prime}\right)$ \\
\hline Ibov63R & AGGAACCCTCATTATGCTGTC \\
\hline Ibov64F & CTAGGTCATGTAAATCCCCTGG \\
\hline Ibov64R & CAGCACCTTCTCCATACACTG \\
\hline Ibov65F & GGTCCTTGCATAAATGTATAGAGC \\
\hline Ibov65R & GTCCTGGGATCCTGGAATTG \\
\hline Ibov66F & CTGGGTCCTGATTGGTCACT \\
\hline Ibov66R & GGTGCAGAGCACAAAAATCA \\
\hline Ibov67F & TTCTCATGTCTCCCTCTGGG \\
\hline Ibov67R & AACCTCCCCTGTGTCTCGTT \\
\hline Ibov70F & CCAATATTTTGGGGGATCTCT \\
\hline Ibov70R & GATGGAGATGGGGAGTGGTA \\
\hline Ibov71F & TCCTTGCCTTCATGGGATAG \\
\hline Ibov71R & GACCTGCCCTTCCTTACCTC \\
\hline Ibov $72 \mathrm{~F}$ & TTTGTTTGGCATTCAGAGCA \\
\hline Ibov72R & CGAAGTGTTTGACTCCGGTT \\
\hline Ibov73F & GGCTTTTGTAACCTCGCTTG \\
\hline Ibov73R & TCTCTCTCTGGGAACAGCGT \\
\hline Ibov74F & AGCTTTTCCCAACAGCTTCA \\
\hline Ibov74R & TCTGGGCTTTTAGCTGCATT \\
\hline Ibov75F & GAAAGTTGCAGGCAACCACT \\
\hline Ibov75R & TGAACTTGCCATGTGCTCTC \\
\hline Ibov $76 \mathrm{~F}$ & TGTGTGAGGATTTAATAGGGCA \\
\hline Ibov76R & TGCCTGAATCTCTGGTGTCA \\
\hline Ibov $77 \mathrm{~F}$ & GCATGGTGGTTTGGTTTTCT \\
\hline Ibov77R & ACCATCTTTGGGGAAAAAGC \\
\hline Ibov78F & GGGATCCTCAAGATCATGTCA \\
\hline Ibov78R & TGTGATCTAAATTTATGTGGCATT \\
\hline Ibov79F & CCAAACGATTTTCAAGAAAGG \\
\hline Ibov79R & CTCTGTGTCCCTTTATGTGGG \\
\hline Ibov80F & TGGGAGCTCACCAAAAGAAT \\
\hline Ibov80R & TTGCTTCTATGGCAAAGCCT \\
\hline Ibov81F & AACCACCCAAATCCCATGTA \\
\hline Ibov81R & AACACAAACTCTGTTTTAAATCTTACG \\
\hline Ibov82F & TCGGGACCTCTTCATTTGAG \\
\hline Ibov82R & TGTTTGTGCAATTTGAGCATT \\
\hline Ibov83F & TGCTACCCTGGTAACCAACA \\
\hline Ibov83R & GAAAGGAAATTAGGGGCCAG \\
\hline Ibov84F & CCCCAACAATTCTGTCCATT \\
\hline Ibov84R & TGTCAAGCATTTCCAAAGCA \\
\hline Ibov85F & TTCTACCTACATTGCCCCCA \\
\hline Ibov85R & TGTCAAGCATTTCCAAAGCA \\
\hline Ibov86F & TTGCCATGTAAATTTCCCAGA \\
\hline Ibov86R & CTCGAATACTGTTCAGCGCA \\
\hline Ibov87F & CATAACTGAGAGCCATTGGGA \\
\hline Ibov87R & GGGATAACAGGCATGACACC \\
\hline Ibov88F & TCAAACCATCGTGTGAAAGAA \\
\hline Ibov88R & TGAAGCAGAGGACTCCATGA \\
\hline Ibov89F & CTCACCACAACCAGTTCCCT \\
\hline Ibov89R & GGTAGCCTTTTGGTTAGCCC \\
\hline Ibov90F & GGGGTAGAAGGCCTGAAACT \\
\hline Ibov90R & CGACCCAAGAGCATTTGTTT \\
\hline Ibov91F & TTCTCATGTCTCCCTCTGGG \\
\hline Ibov91R & CTCCCCTGTGTCTCGTTTTT \\
\hline Ibov92F & CCTAAAGCCATCCAAAGAGC \\
\hline Ibov92R & ACCGACTCGAAGGACATTTG \\
\hline Ibov93F & AATGCCAGTTTGGCAGTCAG \\
\hline Ibov93R & ATCAGTGAGCCCCAGACAAC \\
\hline Ibov94F & GCTCCACCCAGGGACTAAA \\
\hline Ibov94R & GGATCAGGATGAGAACCCAA \\
\hline Ibov95F & AAGACCAGACCCATCTGCAC \\
\hline Ibov95R & TTCAGCATGTGCTTCAAAGG \\
\hline Ibov96F & GCTGTCCAAGGGACTTTCAA \\
\hline Ibov96R & ATCCATGATTGGTCCTGCTC \\
\hline
\end{tabular}




\section{Analysis of indel polymorphisms in silver-stained high-resolution polyacrylamide gels}

To make indel analysis less expensive and independent of sophisticated equipment, we successfully adapted our primers and multiplex systems for genotyping in long thin denaturing polyacrylamide gels with silver staining, exactly as described previously (Pena and Pena, 2012). These gels can be easily run and fully automated with a simple large scanner and commercial software as described by Pena and Pena (2012).

\section{RESULTS AND DISCUSSION}

\section{Identification of polymorphic loci and estimation of allele frequencies}

We initially tested 94 indel polymorphisms with two or more base pair differences between alleles using as samples two DNA pools of $60 \mathrm{Zebu}$ and 60 taurine animals, respectively. From the amplification of these indels, we identified 50 indels that showed two or more identifiable alleles, i.e., that were polymorphic in B. taurus indicus (Table 3).

We then amplified each locus using individual samples of 70 Zebu and 70 taurine animals to estimate the frequency of each allele (Table 3 ) to select the 22 indels with heterozygosity closest to $50 \%$ (bold type in Table 3 ). The allelic sizes of the chosen indels are shown in Table 4.

Table 3. Allele frequencies of polymorphic insertion/deletion loci identified in Bos taurus indicus.

\begin{tabular}{|c|c|c|c|c|c|c|c|}
\hline Locus & Allele 1 & Allele 2 & Allele 3 & Locus & Allele 1 & Allele 2 & Allele 3 \\
\hline Ibov01 & 0.24 & 0.76 & - & Ibov47 & 0.14 & 0.86 & - \\
\hline Ibov02 & 0.87 & 0.13 & - & Ibov50 & 0.36 & 0.64 & - \\
\hline Ibov03 & 0.48 & 0.52 & - & Ibov52 & 0.21 & 0.79 & - \\
\hline Ibov05 & 0.51 & 0.49 & - & Ibov55 & 0.09 & 0.91 & - \\
\hline Ibov08 & 0.20 & 0.80 & - & Ibov56 & 0.84 & 0.16 & - \\
\hline Ibov10 & 0.20 & 0.39 & 0.41 & Ibov60 & 0.71 & 0.29 & - \\
\hline Ibov13 & 0.07 & 0.93 & - & Ibov61 & 0.39 & 0.61 & - \\
\hline Ibov15 & 0.61 & 0.39 & - & Ibov62 & 0.26 & 0.24 & 0.50 \\
\hline Ibov18 & 0.32 & 0.68 & - & Ibov66 & 0.69 & 0.31 & \\
\hline Ibov22 & 0.10 & 0.90 & - & Ibov67 & 0.26 & 0.44 & 0.30 \\
\hline Ibov27 & 0.42 & 0.58 & - & Ibov72 & 0.54 & 0.46 & - \\
\hline Ibov30 & 0.90 & 0.10 & - & Ibov73 & 0.25 & 0.37 & 0.38 \\
\hline Ibov31 & 0.48 & 0.52 & - & Ibov74 & 0.52 & 0.48 & - \\
\hline Ibov32 & 0.12 & 0.88 & - & Ibov76 & 0.38 & 0.62 & - \\
\hline Ibov34 & 0.81 & 0.19 & - & Ibov77 & 0.78 & 0.22 & - \\
\hline Ibov37 & 0.52 & 0.48 & - & Ibov78 & 0.44 & 0.56 & - \\
\hline Ibov38 & 0.76 & 0.24 & - & Ibov79 & 0.50 & 0.50 & - \\
\hline Ibov39 & 0.55 & 0.45 & - & Ibov80 & 0.28 & 0.72 & - \\
\hline Ibov40 & 0.50 & 0.50 & - & Ibov83 & 0.33 & 0.35 & 0.32 \\
\hline Ibov41 & 0.76 & 0.24 & - & Ibov87 & 0.25 & 0.46 & 0.29 \\
\hline Ibov42 & 0.53 & 0.47 & - & Ibov89 & 0.80 & 0.20 & - \\
\hline Ibov43 & 0.73 & 0.27 & - & Ibov90 & 0.89 & 0.11 & - \\
\hline Ibov44 & 0.30 & 0.70 & - & Ibov91 & 0.23 & 0.39 & 0.38 \\
\hline Ibov45 & 0.14 & 0.61 & 0.25 & Ibov93 & 0.67 & 0.33 & - \\
\hline Ibov46 & 0.54 & 0.46 & - & Ibov96 & 0.53 & 0.47 & - \\
\hline
\end{tabular}

The 22 indels with heterozygozity closest to $50 \%$ (bold type) were selected to be included in the multiplex sets. 


\begin{tabular}{|c|c|c|c|c|c|}
\hline \multicolumn{3}{|c|}{ Multiplex 1} & \multicolumn{3}{|c|}{ Multiplex 2} \\
\hline Indel & Allele & Size (bp) & Indel & Allele & Size (bp) \\
\hline \multirow[t]{2}{*}{ Ibov01 } & 1 & 236 & Ibov41 & 1 & 269 \\
\hline & 2 & 238 & & 2 & 274 \\
\hline \multirow[t]{2}{*}{ Ibov15 } & 1 & 129 & Ibov45 & 1 & 212 \\
\hline & 2 & 133 & & 2 & 214 \\
\hline \multirow{2}{*}{ Ibov18 } & 1 & 161 & & 3 & 218 \\
\hline & 2 & 165 & Ibov67 & 1 & 127 \\
\hline \multirow{2}{*}{ Ibov27 } & 1 & 208 & & 2 & 129 \\
\hline & 2 & 215 & Ibov72 & 1 & 159 \\
\hline \multirow{2}{*}{ Ibov37 } & 1 & 176 & & 2 & 161 \\
\hline & 2 & 191 & Ibov74 & 1 & 136 \\
\hline \multirow[t]{2}{*}{ Ibov39 } & 1 & 279 & & 2 & 138 \\
\hline & 2 & 298 & & 3 & 185 \\
\hline \multirow[t]{2}{*}{ Ibov 40} & 1 & 101 & & 4 & 187 \\
\hline & 2 & 124 & Ibov76 & 1 & 117 \\
\hline \multirow[t]{2}{*}{ Ibov42 } & 1 & 117 & & 2 & 119 \\
\hline & 2 & 120 & Ibov78 & 1 & 105 \\
\hline \multirow[t]{2}{*}{ Ibov52 } & 1 & 75 & & 2 & 107 \\
\hline & 2 & 87 & Ibov83 & 1 & 94 \\
\hline \multirow{2}{*}{ Ibov60 } & 1 & 103 & & 2 & 96 \\
\hline & 2 & 106 & & 3 & 98 \\
\hline \multirow{2}{*}{ Ibov79 } & 1 & 93 & Ibov91 & 1 & 151 \\
\hline & 2 & 95 & & 2 & 152 \\
\hline \multirow{3}{*}{ Ibov87 } & 1 & 150 & & 3 & 153 \\
\hline & 2 & 152 & Ibov93 & 1 & 144 \\
\hline & & & & 2 & 146 \\
\hline
\end{tabular}

Having selected the indels with the highest heterozygosity in B. taurus indicus, the next step was to be able to multiplex them. Accordingly, new primers were designed using the muPlex software (Rachlin et al., 2005) (Table 5) to achieve multiplexing of 11 indels for use in each of two PCR analyses (Table 6). An example of multiplex PCR amplification and resolution by capillary electrophoresis and fluorescence detection in the MegaBACE 1000 automatic DNA sequencer is shown in Figure 1. The system of analysis of bovine DNA samples with these two multiplexes was called MULTINDELS-BOV.

\section{Calculation of match probabilities}

The probability of random match (match probability) can be easily calculated using the multiplication rule from the frequencies of the 22 indel markers (National Research Council, 1996). In our case, the probability of random match of the two multiplexes for analysis in an automated sequencer was estimated at $1.12 \times 10^{-8}$ ( 1 in 89 million) for $B$. taurus indicus and $1.60 \times 10^{-6}$ ( 1 in 620 thousand) for $B$. taurus taurus. These very low match probabilities make MULTINDELS-BOV well suited to serve as a simple and effective traceback method for cattle in Brazil.

\section{Indel panel to differentiate Bos subspecies}

Using the data obtained from the indel genotyping, the Structure program was used to analyze the capacity of the indel multiplexes (MULTINDELS-BOV) to discriminate individual animals according to subspecies. Thirty-three animals from each of the subspecies were 


\section{Table 5. Multiplex PCR primers.}

\begin{tabular}{|c|c|}
\hline Primer name & Primer sequence $\left(5^{\prime}-3^{\prime}\right)$ \\
\hline Ibov03mF & GTGAGGAGAGAATTGCTTCTA \\
\hline Ibov03mR & TGCCCTCACTGTCCTATC \\
\hline Ibov05mF & TCCTGTAGCAGCCATACTT \\
\hline Ibov05mR & CCAATGAGACGTGACAAGA \\
\hline Ibov08mF & CAGGCTGTGGAAATCTAATC \\
\hline Ibov08mR & CAAGGAAATGTGTGGCTT \\
\hline Ibov $10 \mathrm{mF}$ & ATAGGAATGAGTGGCCATA \\
\hline Ibov $10 \mathrm{mR}$ & CCCATTCACTGAAACAAAC \\
\hline Ibov $15 \mathrm{mF}$ & GGAAGAGCAATAGCCCAG \\
\hline Ibov $15 \mathrm{mR}$ & TTGTTGCTTATACCATCATCA \\
\hline Ibov $27 \mathrm{mF}$ & GATCCATGCTTCTCTGTTATT \\
\hline Ibov $27 \mathrm{mR}$ & GCAATCCTTGTGTGAAGTAGT \\
\hline Ibov $30 \mathrm{mF}$ & AAGGAACCAATTGTTACGAC \\
\hline Ibov $30 \mathrm{mR}$ & GCTGCTTGGCTTATAGGAT \\
\hline Ibov $31 \mathrm{mF}$ & CACGGCCATCATAGGTAG \\
\hline Ibov $31 \mathrm{mR}$ & TGGAGTAATTCCGCATCA \\
\hline Ibov $34 \mathrm{mF}$ & TTCACACTGCACTTCAATATC \\
\hline Ibov $34 \mathrm{mR}$ & TTTTCAAGATCTGAAGGAATTGAAA \\
\hline Ibov $37 \mathrm{mF}$ & CССТACTCCCTGGAGTCT \\
\hline Ibov $37 \mathrm{mR}$ & TGAGGACAAACTCTGGCTA \\
\hline Ibov $38 \mathrm{mF}$ & GGTGGACAAACACCAAAT \\
\hline Ibov $38 \mathrm{mR}$ & AACTCTTCACCTGGATCTAGA \\
\hline Ibov $39 \mathrm{mF}$ & CCCTGCTCCATATATACCTG \\
\hline Ibov $39 \mathrm{mR}$ & AAATACCAAGCAATCCTGAG \\
\hline Ibov $40 \mathrm{mF}$ & CGTGTGTGTGTCCATAATATC \\
\hline Ibov $40 \mathrm{mR}$ & CAAGAAACTCAACATGGGAG \\
\hline Ibov $42 \mathrm{mF}$ & ACATGGGAGGTTCACAATA \\
\hline Ibov $42 \mathrm{mR}$ & TCACCTGTATTTACAGCCG \\
\hline Ibov $44 \mathrm{mF}$ & CAGATACCTCATGAAAGAGAAA \\
\hline Ibov $44 \mathrm{mR}$ & TTGACTCTGCCTCTAGGTAAGAGT \\
\hline Ibov $45 \mathrm{mF}$ & GTAAATGGTTCGCTCTGAC \\
\hline Ibov $45 \mathrm{mR}$ & AGCACTGAATGCTGTAACTG \\
\hline Ibov $46 \mathrm{mF}$ & TTGACCCATTATAAAGCAAA \\
\hline Ibov $46 \mathrm{mR}$ & CAATTCAAGCAAAGTGCTG \\
\hline Ibov $50 \mathrm{mF}$ & GGTTACTGAGGCCTATGAAA \\
\hline Ibov $50 \mathrm{mR}$ & TCACAACTGAAATAGCATTGTA \\
\hline Ibov $52 \mathrm{mF}$ & TGGTTAGGAGAGCTCCATT \\
\hline Ibov $52 \mathrm{mR}$ & CCTACACACCACCCACATA \\
\hline Ibov60mF & CTCTTCTTGTTTCTTTCTTTCT \\
\hline Ibov $60 \mathrm{mR}$ & TATACCTGTGGCGGATTC \\
\hline Ibov $65 \mathrm{mF}$ & AGGACCCTGAGTCGTCTG \\
\hline Ibov $65 \mathrm{mR}$ & AGGAGGTTCTAAATTATCCAT \\
\hline Ibov66mF & GAGATAGCAGCGAGCCAATC \\
\hline Ibov66mR & GGTGCAGAGCACAAAAATCA \\
\hline Ibov $72 \mathrm{mF}$ & TTGGCATTCAGAGCATAAT \\
\hline Ibov $72 \mathrm{mR}$ & CAAAGGCATGTGTTTCTAGA \\
\hline Ibov $74 \mathrm{mF}$ & CCAAGGACAACTCCACAG \\
\hline Ibov $74 \mathrm{mR}$ & ATCGACTTCTCTCTCTCCTG \\
\hline Ibov $76 \mathrm{mF}$ & GCTATAGTTTCATGACACAGTAA \\
\hline Ibov $76 \mathrm{mR}$ & CTTCTCATTTATCAAGGCAC \\
\hline Ibov $78 \mathrm{mF}$ & CCTACTTCATGCTTGTGTGA \\
\hline Ibov $78 \mathrm{mR}$ & ACGTCATCATCAACAACAAA \\
\hline Ibov $79 \mathrm{mF}$ & AGAAGCCACTAAATACTTTCTTA \\
\hline Ibov $79 \mathrm{mR}$ & TCСTTCTTTATGTGGGTACA \\
\hline Ibov $80 \mathrm{mF}$ & TCAAAAATGCTACTGACATGGAA \\
\hline Ibov $80 \mathrm{mR}$ & TGGCTTTGTCCTGTCTTTTG \\
\hline Ibov $83 \mathrm{mF}$ & CAACTATACATCGCACACAAA \\
\hline Ibov $83 \mathrm{mR}$ & CTGCCTCCTCGTCTTCTA \\
\hline Ibov $87 \mathrm{mF}$ & AACCATCGTGTGAAAGAATA \\
\hline Ibov $87 \mathrm{mR}$ & GCAAGGAAACAGAACTGATC \\
\hline Ibov $91 \mathrm{mF}$ & GGAGAGACTTGAATCTGAATAC \\
\hline Ibov91mR & ACAAGGTCCTATTGTATAGCAC \\
\hline Ibov $93 \mathrm{mF}$ & TGGCTTGATTCAATTTCTAC \\
\hline Ibov93mR & GCTACATCTTGGAATGGAA \\
\hline Ibov $96 \mathrm{mF}$ & ATCAGTCCTCCAATGAATATT \\
\hline Ibov $96 \mathrm{mR}$ & TTCAGTGGTGGTCTACCTG \\
\hline
\end{tabular}


analyzed, under the premise of the existence of two populations $(k=2)$. The results showed that MULTINDELS-BOV is able to absolutely differentiate the Zebu animals from the taurine animals as shown in Figure 2.

\begin{tabular}{lc} 
Table 6. Distribution of the indel loci in the multiplex PCRs. \\
\hline Multiplex reaction 1 & Multiplex reaction 2 \\
\hline Ibov01 & Ibov41 \\
Ibov15 & Ibov67 \\
Ibov18 & Ibov72 \\
Ibov27 & Ibov74 \\
Ibov37 & Ibov76 \\
Ibov39 & Ibov78 \\
Ibov40 & Ibov79 \\
Ibov42 & Ibov83 \\
Ibov45 & Ibov87 \\
Ibov52 & Ibov91 \\
Ibov60 & Ibov93 \\
\hline
\end{tabular}

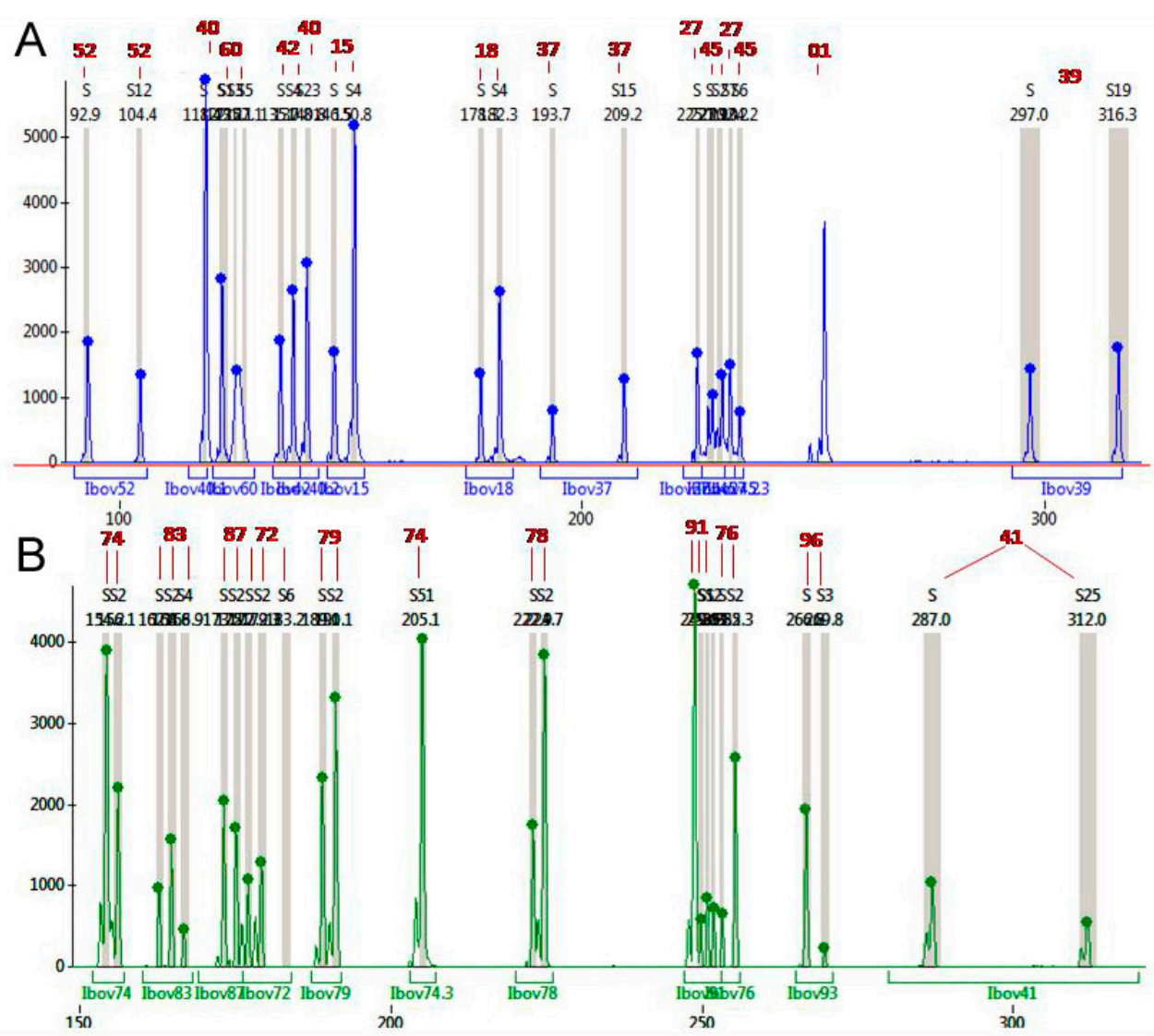

Figure 1. Allelic profile of the two multiplex amplifications using a DNA pool of 60 Bos taurus indicus animals as sample, showing all alleles. A. Multiplex 1; B. Multiplex 2. 


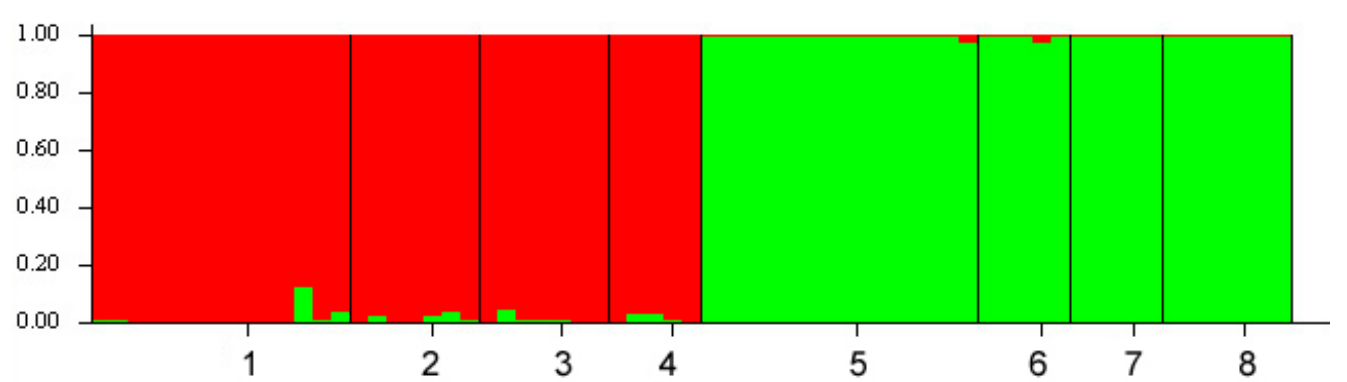

Figure 2. Bar plot generated in the Structure program. The red color designates Zebu ancestry and the numbers 1-4 indicate the races: $1=$ Nellore, $2=$ Gyr, $3=$ Guzerat, and $4=$ Brahman. The green color designates taurine ancestry and the numbers 5-8 indicate the races: $4=$ Holstein, $5=$ Jersey, $6=$ Hereford, and $7=$ Angus.

\section{Analysis of indel polymorphisms in silver-stained high-resolution polyacrylamide gels}

Developing countries such as Brazil are technological mosaics. While some regions of the country have state-of-the-art laboratories, other poorer regions may lack the budget for the large expenses involved in purchasing and operating equipment such as fluorescent DNA analyzers. In such "low-tech" laboratories, the simplicity of running and analyzing the simple diallelic indel multiplexes would be especially helpful.

Thus, we successfully developed a parallel multiplex system (SILVER MULTINDELSBOV) for genotyping the 22-indel panel in long thin denaturing polyacrylamide gels with silver staining, which can be highly useful as a low-cost adjunct to the more technologically complex fluorescent typing of the same loci in automatic DNA sequencers. For that, it was necessary to design special PCR primers, which are listed in Table 7. The analysis using polyacrylamide gel electrophoresis allowed good discrimination of the amplicons (Figure 3).

The great advantage of this new system of typing is its low cost. Besides a PCR thermocycler, all the special laboratory equipment needed is an electrophoresis DNA sequencing apparatus for long thin denaturing gels, a high wattage power supply, and a large flat scanner. The total cost for this equipment is less than US $\$ 10,000$. The unlabeled primers and all other supplies are very inexpensive, so genotyping of an individual animal will cost less than US\$15.00.

\section{CONCLUSION}

Through the use of multiplexed indel polymorphisms, MULTINDELS-BOV proved to be advantageous for cattle traceback and provided excellent results as shown above. The method is simple, rapid, and readily automatable, since in the vast majority of cases, there are only two alleles at each locus. Moreover, the size of the amplification products can be reduced, facilitating the analysis of degraded DNA samples.

Thus, we feel that the MULTINDELS-BOV indel panel that we developed should be capable of providing traceability of cattle in Brazil, generating greater import market confidence, applications in veterinary forensics in the identification of stolen animals (even if they have been slaughtered), and as a bonus, the capacity to estimate the relatedness and to identify the subspecies of cattle using simple admixture genetics (Bicalho et al., 2006). 


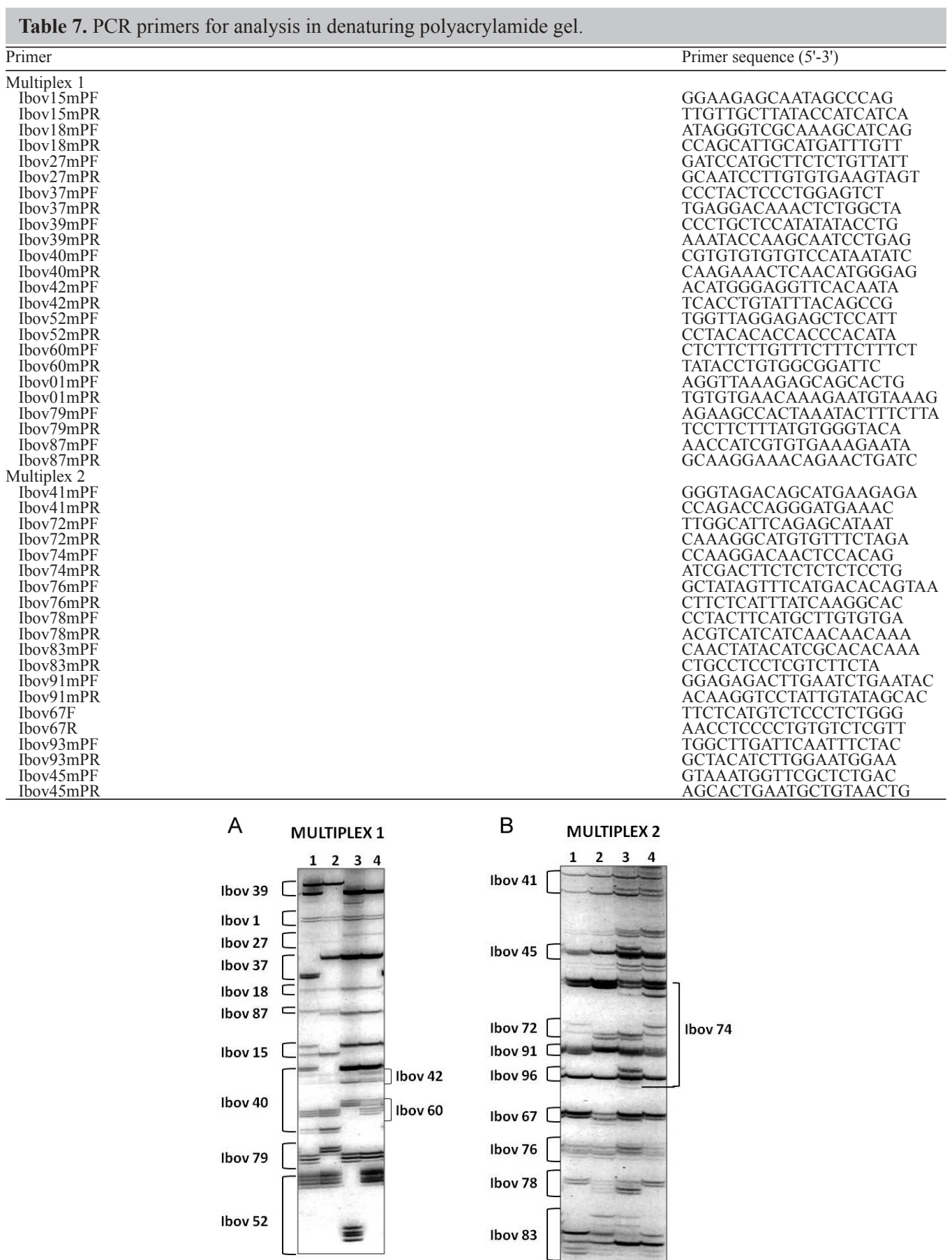

Figure 3. Profile of indels (Multiplex 1 and Multiplex 2) in a high-resolution $(40 \mathrm{~cm})$ denaturing polyacrylamide gel stained with silver nitrate. A. Multiplex 1: lanes 1 and $2=$ Nelore animal; lane $3=$ Jersey animal; lane $4=$ Holstein animal. B. Multiplex 2: lanes 1 and $2=$ Nelore animal; lane $3=$ Jersey animal; lane $4=$ Holstein animal. 


\section{ACKNOWLEDGMENTS}

Research supported by Fundação de Amparo à Pesquisa do Estado de Minas Gerais (FAPEMIG) and Conselho Nacional de Desenvolvimento Científico e Tecnológico (CNPq) of Brazil.

\section{REFERENCES}

Allen AR, Taylor M, McKeown B, Curry AI, et al. (2010). Compilation of a panel of informative single nucleotide polymorphisms for bovine identification in the Northern Irish cattle population. BMC Genet. 11-5.

Bastos-Rodrigues L, Pimenta JR and Pena SDJ (2006). The genetic structure of human populations studied through short insertion-deletion polymorphisms. Ann. Hum. Genet. 70: 658-665.

Bicalho HM, Pimenta CG, Mendes IK, Pena HB, et al. (2006). Determination of ancestral proportions in synthetic bovine breeds using commonly employed microsatellite markers. Genet. Mol. Res. 5: 432-437.

Bowling MB, Pendell DL, Morris DL, Yoon Y, et al. (2008). Identification and traceability of cattle in selected countries outside of North America. PAS 24: 287-294.

Cargill EJ and Womack JE (2007). Detection of polymorphisms in bovine toll-like receptors 3, 7, 8, and 9. Genomics 89: 745-755.

FAOSTAT (2011). Production: Live Animals, Cattle, Brazil. Available at [http://faostat3.fao.org/home/index. html\#VISUALIZE]. Accessed January 7, 2014.

Ferraz AL, Bortolossi JC, Curi RA, Ferro MI, et al. (2006). Identification and characterization of polymorphisms within the 5 ' flanking region, first exon and part of first intron of bovine GH gene. J. Anim. Breed. Genet. 123: 208-212.

Groeneveld LF, Lenstra JA, Eding H, Toro MA, et al. (2010). Genetic diversity in farm animals - a review. Anim. Genet. 41 (Suppl 1): 6-31.

Hills D, Schlaepfer J, Comincini S, MacLean I, et al. (2003). Sequence variation in the bovine and ovine PRNP genes. Anim. Genet. 34: 183-190.

Hoashi S, Ashida N, Ohsaki H, Utsugi T, et al. (2007). Genotype of bovine sterol regulatory element binding protein-1 (SREBP-1) is associated with fatty acid composition in Japanese black cattle. Mamm. Genome 18: 880-886.

Karniol B, Shirak A, Baruch E, Singrün C, et al. (2009). Development of a 25-plex SNP assay for traceability in cattle. Anim. Genet. 40: 353-356.

Kumar P, Freeman AR, Loftus RT and Gaillard C (2003). Admixture analysis of South Asian cattle. Heredity 91: 43-50.

Lima VMB, Bornstein CT and Cukierman HL (2006). The Brazilian bovine traceability system - a critical appraisal. Estud. Soc. Agric. 14: 49-87.

Nakatsu Y, Yamada K, Ueda J, Onogi A, et al. (2004). Genetic polymorphisms and antiviral activity in the bovine MX1 gene. Anim. Genet. 35: 182-187.

National Research Council (1996). The Evaluation of Forensic Evidence. National Academy Press, Washington, 31.

Orrù L, Catillo G, Napolitano F, De Matteis G, et al. (2009). Characterization of a SNPs panel for meat traceability in six cattle breeds. Food Control 20: 856-860.

Pascoal A, Prado M, Calo P, Cepeda A, et al. (2005). Detection of bovine DNA in raw and heat-processed foodstuffs, commercial foods and specific risk materials by a novel specific polymerase chain reaction method. Eur. Food Res. Technol. 220: 444-450.

Pena HB and Pena SD (2012). Automated genotyping of a highly informative panel of 40 short insertion-deletion polymorphisms resolved in polyacrylamide gels for forensic identification and kinship analysis. Transf. Med. Hemother. 39: 211-216.

Pritchard JK, Stephens M and Donnelly P (2000). Inference of population structure using multilocus genotype data. Genetics 155: 945-959.

Rachlin J, Ding CM, Cantor C and Kasif S (2005). muPlex: A multi-objective approach to multiplex pcr assay design. Nucleic Acids Res. 33: W544-W547.

Sander P, Hamann H, Pfeiffer I, Wemheuer W, et al. (2004). Analysis of sequence variability of the bovine prion protein gene (PRNP) in German cattle breeds. Neurogenetics 5: 19-25.

Sasazaki S, Mutoha H, Tsurifunea K and Mannena H (2007): Development of DNA markers for discrimination between domestic and imported beef. Meat Sci. 77: 161-166.

Seabury CM, Cargill EJ and Womack JE (2007). Sequence variability and protein domain architectures for bovine Tolllike receptors 1, 5, and 10. Genomics 90: 502-515. 
Siadkowska E, Zwierzchowski L, Oprządek J, Strzałkowska N, et al. (2006). Effect of polymorphism in IGF-1 gene on production traits in Polish Holstein-Friesian cattle. Anim. Sci. Pap. Rep. 24: 225-237.

Shackell GH, Mathias HC, Cave VM and Dodds KG (2005). Evaluation of microsatellites as a potential tool for product tracing of ground beef mixtures. Meat Sci. 70: 337-345.

Ye J, Coulouris G, Zaretskaya I, Cutcutache I, et al. (2012). Primer-BLAST: A tool to design target-specific primers for polymerase chain reaction. BMC Bioinformatics 13: 134. 